\title{
Rational Design of Original Materials for the Electrocatalytic \\ Hydrogenation Reactions: Concept, Preparation, Characterization and Theoretical Analysis
}

\author{
Geneviève Saint-Pierre, Alexandre Chagnes, Nicolas Alexandre-Bouchard, \\ Pierre Harvey, Louis Brossard, and Hugues Ménard
}

\section{SUPPORTING INFORMATION}

\section{Table of contents}

Scheme S1: Codes used in these computations and for the Tables S1-S4.

$\mathrm{S} 2$

Table S1: Atomic charges (in unit of e) calculated on $\mathrm{SiO}_{2}-\mathrm{C}_{1}$

for a small block $\left(\mathrm{U}_{1}\right)$ and a bigger block $\left(\mathrm{U}_{2}\right)$.

Table S2 : Atomic charges (in unity of e) on $\mathrm{SiO}_{2}-\mathrm{C}_{8}$ material. $\quad \mathrm{S} 4$

Table S3 : Atomic charges (in unity of e) on $\mathrm{SiO}_{2}$-Diol material. S5

Table S4 : Atomic charges (in unity of e) on $\mathrm{SiO}_{2}$-Phenyl material. S6 
Scheme S1: Codes used in these computations and Tables S1-S4.

$\underline{\text { Silicon }}$<smiles>O[Si](O)(O)O</smiles><smiles>C[Si](C)(C)O</smiles><smiles>[B][Si](C)(C)OC</smiles>

\section{$\underline{\text { Oxygen }}$}

01<smiles>C[Si](C)(C)O[Si](C)(C)C</smiles><smiles>C[Si](C)(C)O[O]</smiles>

O3<smiles>C[Si](C)(C)OS(C)(C)P</smiles>

\section{$\underline{\text { Carbon }}$}<smiles>C[Si](C)(C)Cl</smiles>

$\underline{\text { Hydrogen }}$

H1 Si-O-H H3 C-H 
Table S1: Atomic charges (in unit of e) calculated on $\mathrm{SiO}_{2}-\mathrm{C}_{1}$ for a small block $\left(\mathrm{U}_{1}\right)$ and a bigger block $\left(\mathrm{U}_{2}\right)$.

\begin{tabular}{lrrrrrrrrr}
\hline Atom type & $\mathrm{Si} 2$ & $\mathrm{O} 3$ & $\mathrm{O} 1$ & $\mathrm{O} 2$ & $\mathrm{C} 1$ & $\mathrm{Si3}$ & $\mathrm{H} 1$ & $\mathrm{H} 3$ & $\mathrm{Si} 1$ \\
$\mathrm{U}_{1}$ & 1.932 & -0.965 & -0.991 & -0.686 & 0.632 & 1.570 & 0.246 & 0.093 & 2.009 \\
Error & 0.011 & 0.005 & 0.010 & 0.019 & 0.013 & 0.007 & 0.014 & 0.022 & 0.020 \\
$\mathrm{U}_{2}$ & 1.938 & -0.970 & -0.997 & -0.690 & 0.636 & 1.564 & 0.239 & 0.093 & 2.009 \\
Error & 0.010 & 0.011 & 0.011 & 0.025 & 0.010 & 0.032 & 0.014 & 0.013 & 0.011 \\
\hline
\end{tabular}


Table S2 : Atomic charges (in unity of e) on $\mathrm{SiO}_{2}-\mathrm{C}_{8}$ material.

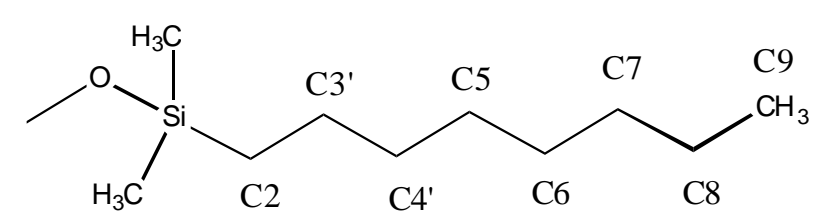

\begin{tabular}{lrrrrrrrr}
\hline Atom & Sil & Si2 & Si3 & O1 & O2 & O3 & H1 & H3 \\
\hline Charge & 1.989 & 1.936 & 1.557 & -0.991 & -0.698 & -0.967 & 0.243 & 0.080 \\
Uncertainties & 0.089 & 0.028 & 0.024 & 0.022 & 0.078 & 0.028 & 0.027 & 0.016 \\
& & & & & & & & \\
\hline
\end{tabular}

\begin{tabular}{lrrrrrrrrr}
\hline Atom & C1 & C2 & C3' & C4' & C5 & C6 & C7 & C8 & C9 \\
\hline Charge & -0.632 & -0.564 & -0.136 & -0.136 & -0.158 & -0.158 & -0.158 & -0.159 & -0.211 \\
Uncertainties & 0.013 & 0.028 & 0.011 & 0.011 & 0.003 & 0.005 & 0.002 & 0.004 & 0.004 \\
& & & & & & & & & \\
\hline
\end{tabular}


Table S3 : Atomic charges (in unity of e) on $\mathrm{SiO}_{2}$-Diol material.

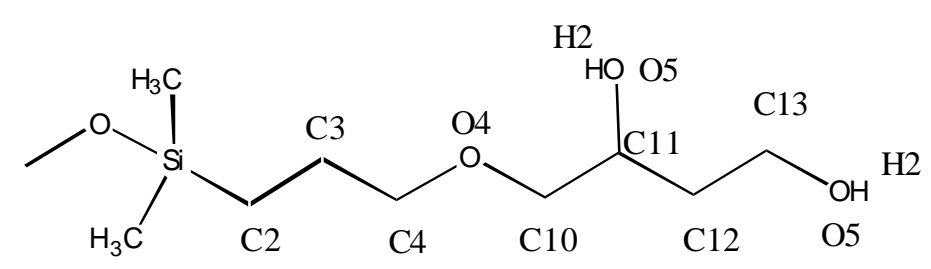

\begin{tabular}{lrrrrrrrrrrr}
\hline Atom & Si1 & $\mathrm{Si2}$ & $\mathrm{Si3}$ & $\mathrm{O} 1$ & $\mathrm{O} 2$ & $\mathrm{O} 3$ & $\mathrm{O} 4$ & $\mathrm{O} 5$ & $\mathrm{H} 1$ & $\mathrm{H} 2$ & $\mathrm{H} 3$ \\
\hline Charge & 2.010 & 1.936 & 1.564 & -0.994 & -0.692 & -0.962 & -0.302 & -0.342 & 0.252 & 0.218 & 0.090 \\
Uncertainties & 0.024 & 0.018 & 0.021 & 0.013 & 0.026 & 0.005 & 0.025 & 0.033 & 0.024 & 0.021 & 0.016 \\
\hline
\end{tabular}

\begin{tabular}{lrrrrrrrr}
\hline Atom & $\mathrm{C} 1$ & $\mathrm{C} 2$ & $\mathrm{C} 3$ & $\mathrm{C} 4$ & $\mathrm{C} 10$ & $\mathrm{C} 11$ & $\mathrm{C} 12$ & $\mathrm{C} 13$ \\
\hline Charge & -0.626 & -0.564 & -0.144 & -0.016 & -0.059 & 0.034 & 0.198 & -0.017 \\
Error & 0.075 & 0.022 & 0.005 & 0.002 & 0.010 & 0.002 & 0.007 & 0.015 \\
\hline
\end{tabular}


Table S4 : Atomic charges (in unity of e) on $\mathrm{SiO}_{2}$-Phenyl material ( $\mathrm{H}_{\mathrm{Ph}}$ : Hydrogens in phenyl).

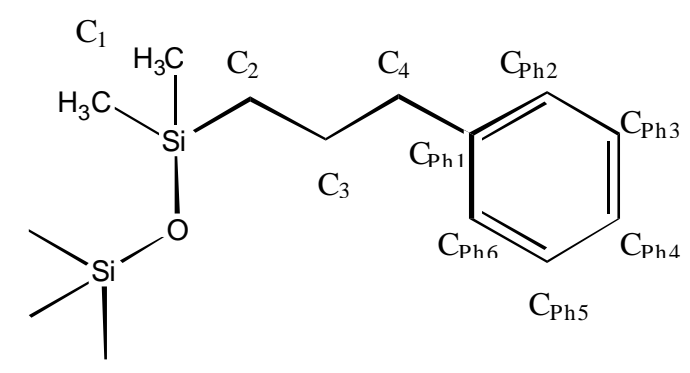

\begin{tabular}{lrrrrrrrrr}
\hline Atom & $\mathrm{Si} 1$ & $\mathrm{Si} 2$ & $\mathrm{Si3}$ & $\mathrm{O} 1$ & $\mathrm{O} 2$ & $\mathrm{O} 3$ & $\mathrm{H} 1$ & $\mathrm{H} 3$ & $\mathrm{H}$ Ph \\
\hline Charge & 2.022 & 1.952 & 1.519 & -0.993 & -0.706 & -0.947 & 0.245 & 0.089 & 0.131 \\
Uncertainties & 0.050 & 0.018 & 0.091 & 0.020 & 0.045 & 0.044 & 0,037 & 0,021 & 0,024 \\
& & & & & & & & & \\
\hline
\end{tabular}

\begin{tabular}{lrrrrrrrrrr}
\hline Atom & $\mathrm{C} 1$ & $\mathrm{C} 2$ & $\mathrm{C} 3$ & $\mathrm{C} 4$ & $\mathrm{C}_{\mathrm{Ph} 1}$ & $\mathrm{C}_{\mathrm{Ph} 2}$ & $\mathrm{C}_{\mathrm{Ph} 3}$ & $\mathrm{C}_{\mathrm{Ph} 4}$ & $\mathrm{C}_{\mathrm{Ph} 5}$ & $\mathrm{C}_{\mathrm{Ph} 6}$ \\
\hline Charge & -0.623 & -0.559 & -0.133 & -0.124 & -0.070 & -0.123 & -0.128 & -0.132 & -0.130 & -0.130 \\
Error & 0.026 & 0.031 & 0.025 & 0.008 & 0.019 & 0.023 & 0.021 & 0.014 & -0.011 & -0.011 \\
& & & & & & & & & & \\
\hline
\end{tabular}

